\title{
Monitoring diagnosis, retention in care and viral load suppression in children testing HIV polymerase chain reaction-positive in two districts in South Africa
}

\author{
F Moyo, ${ }^{1,2,3}$ BSc Hons, MSc; A Haeri Mazanderani, ${ }^{2,4}$ MB ChB; U D Feucht, ${ }^{5,6,7}$ MB ChB, FC Paeds (SA), MMed (Paeds), \\ Dip HIV Man (SA), CAHM, PhD; K Ngoma, ${ }^{8}$ MB BS, MSc; $\mathbf{S}$ Bhardwaj, ${ }^{8}$ MB BS, MD, MPH; M Goosen, ${ }^{2}$ BSc Hons; \\ D Greyling, ${ }^{2}$ BSc Hons; O B Mhlongo, ${ }^{9}$ B Cur; G G Sherman, ${ }^{1,2,10}$ MB BCh, MMed, PhD \\ ${ }^{1}$ Paediatric HIV Diagnostics, Division of Wits Health Consortium, Johannesburg, South Africa \\ ${ }^{2}$ Centre for HIV and STIs, National Institute for Communicable Diseases, National Health Laboratory Service, Johannesburg, South Africa \\ ${ }^{3}$ School of Public Health, Faculty of Health Sciences, University of the Witwatersrand, Johannesburg, South Africa \\ ${ }^{4}$ Department of Medical Virology, Faculty of Health Sciences, University of Pretoria, South Africa \\ ${ }^{5}$ Department of Paediatrics, Faculty of Health Sciences, University of Pretoria, South Africa \\ ${ }^{6}$ Tshwane District Clinical Specialist Team, Tshwane District Health Services, Gauteng Department of Health, South Africa \\ Research Centre for Maternal, Fetal, Newborn and Child Health Care Strategies, University of Pretoria and South African Medical Research \\ Council, Pretoria, South Africa \\ ${ }^{8}$ United Nations Children's Fund (UNICEF), Pretoria, South Africa \\ ${ }^{9}$ National Department of Health, KwaZulu-Natal Province, Pietermaritzburg, South Africa \\ ${ }^{10}$ Department of Paediatrics and Child Health, Faculty of Health Sciences, University of the Witwatersrand, Johannesburg, South Africa
}

Corresponding author: F Moyo (faithmo@nicd.ac.za)

Background. Retention in care is associated with improved virological control and survival among HIV-infected children. However, retention of children in HIV care remains a challenge.

Objectives. To describe, using routine laboratory HIV test data, the retention-in-care and virological outcomes of HIV-infected children aged $<18$ months in two districts in South Africa.

Methods. HIV polymerase chain reaction (PCR)-positive results of children from uMkhanyakude and Tshwane districts in KwaZuluNatal and Gauteng provinces, respectively, tested between April 2015 and May 2016, were extracted from the National Health Laboratory Service's Corporate Data Warehouse (CDW). HIV-related tests (PCR, viral load (VL), CD4+) were documented longitudinally for each child for $\geq 13$ months after the first positive PCR result by manually searching demographics within the CDW, supplemented by an automated patient-linking algorithm. Test sets were linked if two or more demographics (surname, name, date of birth, folder number) matched exactly. Programmatic indicators assessed included age at first positive PCR test, presumed confirmatory test rates, retention in care, and VL suppression at 6 and 12 months.

Results. Ninety-four and 304 children tested HIV PCR-positive in uMkhanyakude and Tshwane, respectively. The median age at diagnosis was 3.6 months (interquartile range (IQR) 1.4 - 7.1) for uMkhanyakude and 2.3 months (IQR 0.1 - 6.7) for Tshwane. In uMkhanyakude, confirmed in utero infections accounted for $18.1 \%$ of transmissions $(n=17)$, compared with $29.6 \%(n=90)$ in Tshwane. Presumed confirmatory test rates following an initial positive PCR result were $77.7 \%$ and $71.7 \%$ for uMkhanyakude and Tshwane, respectively. Within 6 months of starting antiretroviral therapy, 43 children (58.9\%) were lost to follow-up in uMkhanyakude compared with 160 (73.4\%) in Tshwane. Of those retained in care at 6 months with a VL measurement, $15(60.0 \%)$ from uMkhanyakude had a VL $<1000 \mathrm{copies} / \mathrm{mL}$, compared with $24(48.0 \%)$ in Tshwane. For both districts, a third of all HIV PCR-positive children were retained in care at the end of followup, with $29(30.9 \%)$ in uMkhanyakude and 99 (32.5\%) in Tshwane. Of these, $12(41.4 \%)$ had a VL $<1000$ copies/mL in uMkhanyakude compared with $28(28.3 \%)$ in Tshwane.

Conclusions. We demonstrate the value of routine laboratory data in monitoring diagnosis, retention and VL suppression in HIV-infected children. This approach is scalable, can be reported near real-time, is relatively inexpensive to implement, and provides a tool for improving paediatric HIV services until clinical databases can assume this role.

S Afr Med J 2019;109(9):686-692. https://doi.org/10.7196/SAMJ.2019.v109i9.13765

The number of HIV-infected children surviving into adolescence and adulthood has been on an upward trajectory in the era of antiretroviral therapy (ART) in sub-Saharan Africa (SSA). ${ }^{[1]}$ The widespread roll-out of ART in many resource-limited settings has resulted in significant declines in new paediatric infections and AIDS-related deaths. Between 2010 and 2016, the annual number of new HIV infections in children decreased by $56 \%$ in SSA, while AIDS mortality rates decreased by $42 \%{ }^{[2]}$ In spite of these gains, paediatric HIV care continues to lag disproportionately to adult HIV care. In 2016, 54\% of adults eligible for ART received ART globally, compared with $43 \%$ of children. ${ }^{[2]}$ This gap has narrowed as a result of advocacy and mounting pressure for equity across populations living with $\mathrm{HIV}^{[3]}$

As in many resource-limited settings, the South African (SA) paediatric HIV programme has been scaled up remarkably from its humble beginnings in the early 2000 s. Scale-up interventions include 
intensified case-finding initiatives, earlier diagnosis of HIV, and accelerated entry into care. ${ }^{[4]}$ While there is evidence of improvements in HIV diagnosis and access to ART among children, ${ }^{[5,6]}$ retention of children in HIV care remains a challenge.

Horstmann et al..$^{[7]}$ define retention in care as the regular engagement in long-term clinical care of HIV-infected individuals'. Retention in care is associated with virological control and survival among infected children. ${ }^{[7]}$ However, significant drop-offs are observed along the HIV care continuum in many high HIV prevalence settings. ${ }^{[8]}$ Evidence from cohort studies suggests that loss to follow-up (LTFU) rates are higher in children and adolescents compared with adults in HIV care. ${ }^{[9-12]}$ Contributing factors include high mobility of mother-child pairs between facilities and weak systems for tracing and linkage among children. A systematic review of retention outcomes of children in the first year of ART in resourcelimited settings reported that younger age $(<1$ year) was associated with poorer retention among children in HIV care. ${ }^{[13]}$ Similar findings were described in a report of birth HIV diagnosis with ART initiation within the first month of life at a single site in SA. ${ }^{[14]}$ These findings have serious policy implications for SA, where current guidelines recommend birth testing for all HIV-exposed children ${ }^{[15]}$ Previously, the national prevention of mother-to-child transmission (PMTCT) guidelines recommended routine HIV PCR testing at 6 weeks of age for HIV-exposed children. The guidelines were revised in June 2015, recommending universal HIV PCR testing at birth for HIVexposed neonates, followed by repeat tests at 10 weeks and 6 weeks after cessation of breastfeeding for children with previous negative results. ${ }^{[15]}$ At the same time, a second PCR test on a second blood sample replaced a baseline HIV viral load (VL) test as confirmation of HIV-positive status. HIV enzyme-linked immunosorbent assay tests are used to confirm positive HIV PCR tests if the child is $\geq 18$ months old. The guidelines further recommend a VL 6 months after ART initiation to monitor treatment response. If the VL is $<50$ copies $/ \mathrm{mL}$, a VL is repeated after 12 months; if the VL is 50 1000 copies $/ \mathrm{mL}$, a VL is repeated in 6 months; and if the VL is $>1000$ copies $/ \mathrm{mL}$, a repeat VL should be performed in 2 months. ${ }^{[15]}$ However, interpretation and implementation of guidelines is not uniform across or within the nine provinces of SA.

In recent years, complexities in early infant diagnosis have emerged as PMTCT programmes have evolved to include increased number and duration of ART drugs for mothers and infants. This change has resulted in an increase in indeterminate and discordant HIV PCR and VL results that complicate making a definitive diagnosis of HIV in children. ${ }^{[16-18]}$

Longitudinal cohort monitoring of HIV-infected children receiving HIV care is crucial for evaluating programme performance and adapting interventions to reduce gaps along the HIV care continuum. The National Department of Health uses Tier.net, an electronic patient management system for HIV-infected patients accessing HIV treatment in the public sector in SA. However, Tier.net does not yet provide national coverage and relies on data being captured at healthcare facility level after the patient visit. In contrast, laboratory data are captured at local laboratory level on receipt of samples from multiple healthcare facilities, and data capture is a prerequisite before testing can occur. The ratio of healthcare facilities to local laboratories in SA is $>10: 1$. Larger numbers of patients therefore have their data captured by fewer data capturers at laboratory $\mathrm{v}$. facility level, resulting in a more efficient recording system within the laboratory network.

\section{Objectives}

Using a nationally representative routine laboratory HIV test database, to monitor diagnosis, retention in care and VL suppression rates of children testing HIV PCR-positive in two districts in SA.

\section{Methods}

HIV PCR test data of all children from uMkhanyakude and Tshwane districts, in KwaZulu-Natal and Gauteng provinces, respectively, with a positive PCR result between April 2015 and May 2016 were extracted from the Corporate Data Warehouse (CDW) of the National Health Laboratory Service (NHLS). Data extracted included patient name, surname, date of birth, file number, name of health facility, laboratory sample number, test registration date and test result. The HIV PCR test data were de-duplicated per patient, and PCR results for tests performed prior to the study period were searched for to obtain each patient's PCR test result history up to May 2016 and determine the age at the first positive PCR result.

HIV-related tests (VL, HIV enzyme-linked immunosorbent assay (ELISA) and CD4+ counts) from the CDW were documented longitudinally for each child for $\geq 13$ months after the initial positive PCR test, using both an automated patient-linking algorithm and a manual search of the laboratory information system (LIS) and the CDW for laboratory test results. Test sets were linked to a patient if two or more demographics (surname, name, date of birth and folder number) matched exactly. Among neonates, first names and facilities were expected to differ between the birth HIV PCR (performed at $<7$ days of life) and follow-up tests, because many neonates do not have first names at birth and the majority of mother-child pairs are down-referred to a different health facility for routine care after delivery.

Data analysis was censored at LTFU or end of follow-up on 31 May 2017. Programmatic indicators evaluated included:

- Age at HIV diagnosis. Age at first positive HIV PCR result from the LIS and CDW.

- Birth testing coverage. Number of birth HIV PCR tests registered during the study period divided by the estimated number of HIVexposed infants born. The latter was calculated by multiplying live births registered per district (Statistics South Africa, 2015 ${ }^{[19]}$ ) by district antenatal HIV seroprevalence rates (2015 National Antenatal Sentinel HIV \& Syphilis Survey Report ${ }^{[20]}$ ).

- Timing of HIV transmission, as defined in Table 1.

- Presumed confirmatory test. The number of children with an HIV-related test within 3 months of their first positive PCR test was used as a proxy for confirmatory testing of positive HIV status.

Table 1. Definition of timing of HIV transmission in the study cohort

\begin{tabular}{llll}
\hline \multirow{2}{*}{ Timing of transmission } & \multicolumn{2}{c}{ Age at diagnosis } \\
\cline { 2 - 4 } & $<7$ days & $\mathbf{7}$ days $-\leq \mathbf{3}$ months & $>\mathbf{3}$ months \\
\hline In utero & Positive & Not applicable & Not applicable \\
Intrapartum/early postnatal & Negative & Positive & Not applicable \\
Postnatal & Negative & Negative & Positive \\
Unknown (early diagnosis) & Nil & Positive & Not applicable \\
Unknown (later diagnosis) & Nil & Nil & Positive
\end{tabular}


Tests were considered a confirmatory HIV test if children had an HIV PCR, VL or ELISA test, and were counted in that order. In the absence of a confirmatory HIV test, a CD4+ count was considered indicative of HIV-related testing.

- Retained in care at $\mathbf{6}$ months. Number of children with an HIV VL or CD4+ count performed 3 - 9 months after returning for their positive PCR result. According to national guidelines, this coincides with the first visit for VL and CD4+ monitoring after ART initiation. ${ }^{[15]}$

- Retained in care at $\mathbf{1 2}$ months. Number of children retained in care at 6 months, with a subsequent HIV VL or CD4+ count $\geq 9$ months after returning for their PCR result. Children not yet due for their subsequent visit were censored at close of follow-up on 31 May 2017.

The treatment cascade constructed for Tshwane from laboratory test data was compared with a treatment cascade constructed using laboratory test data supplemented by clinical data from Tier.net and a clinical database of a tertiary referral hospital in the district, anticipated to contain records of $\geq 90 \%$ of the district's HIV-infected children on ART. The clinical data included patient demographics, ART start dates, HIV-related test dates, results and date of death to populate the same programmatic indicators as described for the laboratory data. Data analysis censored clinical data at the time of death or LTFU when clinic visits were missed.

\section{Statistical analysis}

Data were imported from Excel 2016 (Microsoft, USA) into Stata 14 (StataCorp, USA) for analysis. Medians and interquartile ranges (IQRs) were used to describe continuous variables, and proportions were used for categorical data. Kaplan-Meier curves were used to explore rates of retention in care, stratified by age of children at HIV diagnosis. The log-rank test was used to assess equality of rates of retention between children's age groups at HIV diagnosis. The Kruskal-Wallis test was used to compare differences of continuous variables between age groups at HIV diagnosis.

\section{Ethical considerations}

This work was approved by the Human Research Ethics Committee of the University of the Witwatersrand (ref. no. M160216). Patient consent was not required, as the study was an audit of routine clinical care within the national HIV surveillance programme. Patient identity was protected by de-identifying data prior to analysis.

\section{Results}

uMkhanyakude District (rural),

\section{KwaZulu-Natal}

A total of 94 children tested HIV PCRpositive during the study period, out of an estimated annual number of 7018 HIVexposed infants with birth testing coverage of $83.2 \%$. The median age at first positive PCR test was 3.6 months (IQR $1.4-7.1$ ) (Table 2). While confirmed in utero infections accounted for $18.1 \%$ of transmissions, $44.7 \%$ of infections were diagnosed after 3 months of age. Of the 94 children with a positive PCR test, $29(30.9 \%)$ were retained in care at the end of the study period, although 7 of them had missed their 6-month blood tests. Of these, $12(41.4 \%)$ had a VL $<1000$ copies $/ \mathrm{mL}$. Seventy-three children (77.7\%) were presumed to have a confirmed HIV diagnosis following their initial positive PCR result (Table 2, Fig. 1A). Sixty children (82.2\%) had a confirmatory HIV test (HIV PCR, VL or

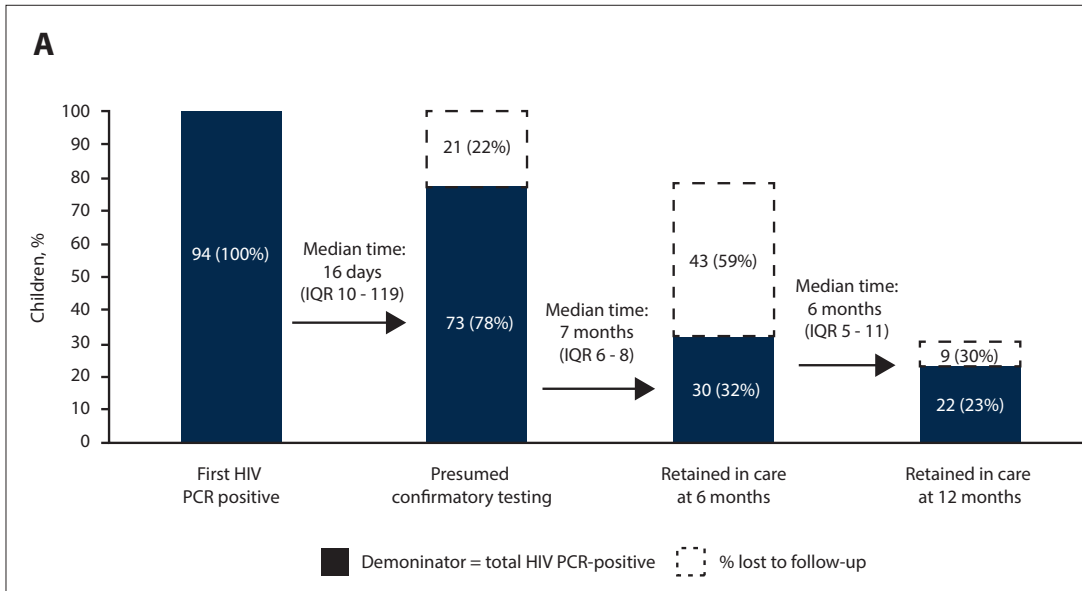

B

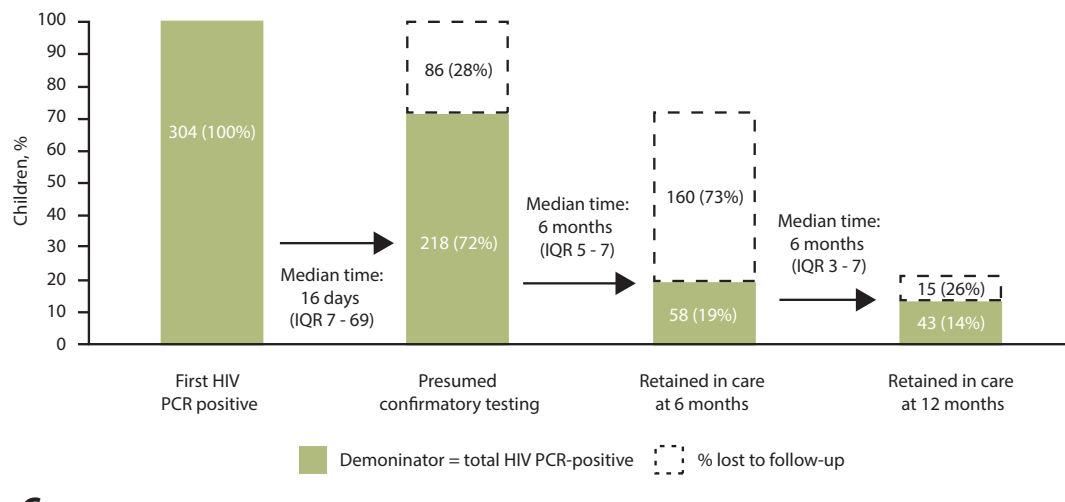

C

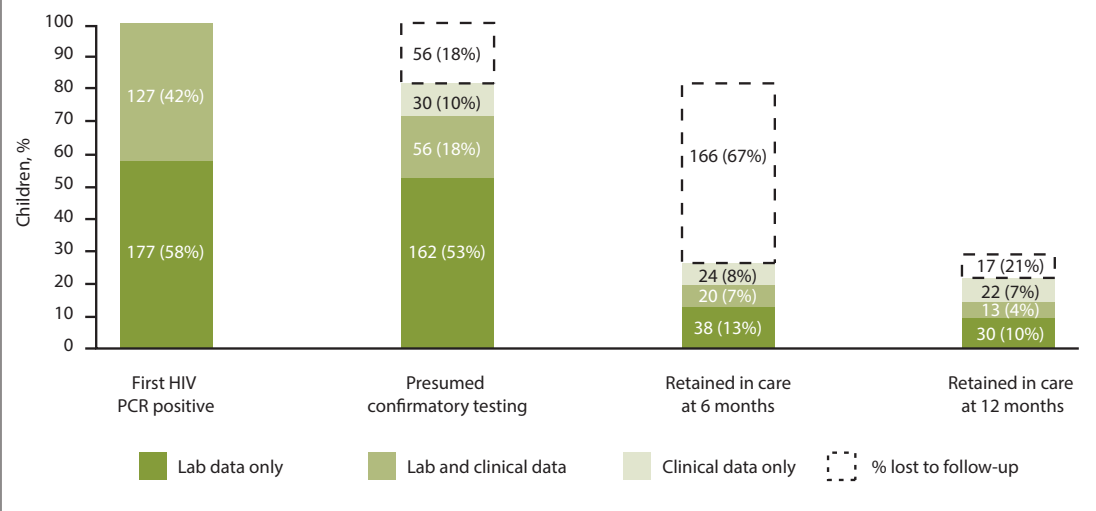

Fig. 1. HIV treatment cascades for children with a first HIV PCR-positive test in (A) uMkhanyakude District and (B) Tshwane District. (C) shows the treatment cascade for the Tshwane cohort using laboratory HIV test data supplemented by clinical data. $(I Q R=$ interquartile range; $P C R=$ polymerase chain reaction; Lab data = data from the National Health Laboratory Service Corporate Data Warehouse; clinical data = data from a hospital database and Tier.net.) 
Table 2. Comparison of the timing and results of HIV tests performed in HIV PCR-positive children from uMkhanyakude and Tshwane districts, South Africa

\begin{tabular}{|c|c|c|}
\hline Variable & uMkhanyakude $(N=94)$ & Tshwane $(N=304)$ \\
\hline \multicolumn{3}{|l|}{ Age at HIV diagnosis } \\
\hline Median (IQR), months & $3.6(1.4-7.1)$ & $2.3(0.1-6.7)$ \\
\hline$<7$ days (birth), $n(\%)$ & $17(18.1)$ & $90(29.6)$ \\
\hline 7 days - $\leq 3$ months, $n(\%)$ & $23(24.5)$ & $85(28.0)$ \\
\hline$>3-<6$ months, $n(\%)$ & $23(24.5)$ & $38(12.5)$ \\
\hline $6-<12$ months, $n(\%)$ & $19(20.2)$ & $54(17.8)$ \\
\hline $12-<18$ months, $n(\%)$ & $11(11.7)$ & $33(10.8)$ \\
\hline Unknown, $n(\%)$ & $1(1.0)$ & $4(1.3)$ \\
\hline Birth testing coverage, $n(\%)$ & 83.2 & 80.3 \\
\hline \multicolumn{3}{|l|}{ Timing of transmission, $n(\%)$} \\
\hline In utero & $17(18.1)$ & $90(29.6)$ \\
\hline Intrapartum/early postnatal & $3(3.2)$ & $16(5.3)$ \\
\hline Postnatal & $11(11.7)$ & $23(7.6)$ \\
\hline Unknown (early diagnosis) & $20(21.3)$ & $73(24.0)$ \\
\hline Unknown (later diagnosis) & $42(44.7)$ & $98(32.2)$ \\
\hline Age at diagnosis not available & $1(1.0)$ & $4(1.3)$ \\
\hline Presumed confirmatory testing, $n(\%)$ & $73(77.7)$ & $218(71.7)$ \\
\hline HIV PCR test on return & $41(56.1)$ & $131(60.0)$ \\
\hline HIV VL test on return & $18(24.7)$ & $61(28.0)$ \\
\hline HIV ELISA on return & $1(1.4)$ & $1(0.5)$ \\
\hline $\mathrm{CD} 4$ count test on return & $13(17.8)$ & $25(11.5)$ \\
\hline \multicolumn{3}{|c|}{ Time to presumed confirmatory testing of initial positive PCR result, median (IQR) } \\
\hline$<7$ days (birth) & $12(10-15)$ & $34(10-77)$ \\
\hline 7 days $-\leq 3$ months & $17(12-36)$ & $17(7-38)$ \\
\hline$>3$ months & $20(11-181)$ & $12(5-34)$ \\
\hline Retained in care at 6 months, $n(\%)$ & $30(41.1)$ & $58(26.6)$ \\
\hline Children with a 6-month VL & $25(83.3)$ & $50(86.2)$ \\
\hline $\mathrm{VL}<1000$ copies $/ \mathrm{mL}$ & $15(60.0)$ & $24(48.0)$ \\
\hline $\mathrm{VL} \geq 1000$ copies $/ \mathrm{mL}$ & $10(40.0)$ & $26(52.0)$ \\
\hline Retained in care at 12 months, $n(\%)$ & $22(73.3)$ & $43(74.1)$ \\
\hline Children with a 12 -month VL & $16(72.7)$ & $31(72.1)$ \\
\hline $\mathrm{VL}<1000$ copies $/ \mathrm{mL}$ & $9(56.2)$ & $18(58.1)$ \\
\hline $\mathrm{VL} \geq 1000$ copies $/ \mathrm{mL}$ & $7(43.8)$ & $13(41.9)$ \\
\hline
\end{tabular}

ELISA). Total follow-up time for these children was 842.8 personmonths, with an overall LTFU rate of 87 per 1000 person-months. Children with positive PCR results at birth had lower LTFU rates than children diagnosed at older ages. The LTFU rate was 72 per 1000 person-months compared with 98 and 87 per 1000 personmonths in children first diagnosed with HIV at ages 7 days $\leq 3$ months and $>3$ months, respectively (log-rank $p$-value $<0.001)$. As age at first positive PCR test increased, the median time to a presumed confirmatory test of a positive PCR result increased, despite lack of statistical evidence for this association $(p=0.276)$ (Table 2).

Of 43 children (58.9\%) who were presumed to have received HIV confirmatory testing but were LTFU at 6 months, 21 (48.8\%) had no further HIV test records, but 22 (51.2\%) had additional HIV-related tests within 3 months, indicating a second visit for HIV-related care after the first positive PCR test. Only 30 children (31.9\%) were retained in care at 6 months (Fig. 1A). Of these, $25(83.3 \%)$ had an HIV VL performed and $15(60.0 \%)$ had a VL $<1000$ copies/mL. Twenty-two children (23.0\%) who were retained in care at 6 months were retained at 12 months.

\section{Tshwane District (urban), Gauteng}

A total of 304 children tested HIV PCR-positive during the study period, out of an estimated annual number of HIV-exposed infants of 14157 with birth testing coverage of $80.3 \%$. The median age at first positive PCR test was 2.3 months (IQR $0.1-6.7$ ). Almost one-third of infections were confirmed as in utero, with $30.3 \%$ of infants having a late diagnosis after 3 months of age (Table 2). Of the 304 children with a positive PCR test, 99 (32.6\%) were retained in care at the end of follow-up, although 41 of them had missed their 6-month blood tests. Of those retained in care, 28 (28.3\%) had a VL $<1000$ copies $/ \mathrm{mL}$.

Two hundred and eighteen children (71.7\%) were presumed to have had a confirmed HIV diagnosis after their initial positive PCR result (Table 2, Fig. 1B). Of these, 193 children $(88.5 \%)$ had a confirmatory HIV test. Total follow-up time was 1562.8 person-months, with an overall LTFU rate of 135 per 1000 person-months, significantly higher than the rate in uMkhanyakude $(p=0.01)$. Children with positive PCR results at birth had lower LTFU rates than children diagnosed at older ages. The LTFU rate was 126 per 1000 personmonths, compared with 130 and 145 per 1000 person-months in children first diagnosed with HIV at ages 7 days $-\leq 3$ months 
Table 3. Implementation of VL monitoring guidelines among study participants who were retained in care and received a VL result at 6 months, by district

\begin{tabular}{|c|c|c|c|c|c|}
\hline District & $\begin{array}{l}\text { VL category at } 6 \text { months } \\
\text { (copies } / \mathrm{mL} \text { ) }\end{array}$ & $n(\%)$ & $\begin{array}{l}\text { LTFU at } \\
12 \text { months }\end{array}$ & $\begin{array}{l}\text { PMTCT policy VL monitoring } \\
\text { requirement }\end{array}$ & $\begin{array}{l}\text { Time (months) to } 12 \text { months } \\
\text { VL, median (IQR) }\end{array}$ \\
\hline uMkhanyakude, & $<50$ & $7(28.0)$ & 0 & Repeat VL in 6 or 12 months & $11.2(5.7-13.2)$ \\
\hline \multirow[t]{2}{*}{ KwaZulu-Natal } & $50-<1000$ & $8(32.0)$ & $3(37.5)$ & Repeat VL in 6 months & $10.6(6.6-11.7)$ \\
\hline & $\geq 1000$ & $10(40.0)$ & $4(40.0)$ & Repeat VL in 2 months & $7.3(4.2-13.5)$ \\
\hline \multirow[t]{3}{*}{ Tshwane, Gauteng } & $<50$ & $20(40.0)$ & $3(15.0)$ & Repeat VL in 6 or 12 months & $7.5(6.0-11.9)$ \\
\hline & $50-<1000$ & $4(8.0)$ & $2(50.0)$ & Repeat VL in 6 months & $7.3(3.8-11.1)$ \\
\hline & $\geq 1000$ & $26(52.0)$ & $7(26.9)$ & Repeat VL in 2 months & $6.6(3.6-10.3)$ \\
\hline
\end{tabular}

and $>3$ months, respectively (log-rank $p$-value $<0.001)$. As age at first positive PCR test increased, the median time to presumed confirmatory testing of a positive PCR result decreased $(p=0.003)$ (Table 2).

Of 160 children (73.4\%) who were presumed to have received HIV confirmatory testing but were LTFU at 6 months, 84 (52.5\%) had no further test records, but $76(47.5 \%)$ had additional HIVrelated tests within 3 months, indicating a second visit for HIVrelated care after the first positive PCR test. Only 58 children $(19.1 \%)$ were retained in care at 6 months. Of these, $50(86.2 \%)$ had an HIV VL performed and $24(48.0 \%)$ had a VL $<1000$ copies/mL. Forty-three children (14.1\%) who were retained in care at 6 months were retained at 12 months.

Intervals between the 6-month VL and the subsequent VL test were measured to assess conformity with VL guidelines (Table 3 ). Regardless of the 6-month VL result, the median time to a subsequent VL test was longer in uMkhanyakude than Tshwane. Children with $\mathrm{VL} \geq 1000$ copies/mL at 6 months returned for their VL at a median of 7.3 months in uMkhanyakude and 6.6 months in Tshwane, instead of the recommended 2 months as per the guidelines.

Fig. 1C depicts the HIV treatment cascade constructed using laboratory HIV test data supplemented by clinical data for Tshwane. The clinical databases documented $41.8 \%$ of the Tshwane cohort in comparison with the laboratory database. Supplementing the laboratory with clinical data increased the presumed confirmatory test rate by $9.9 \%$ $(n=30)$, and decreased LTFU at 6 and 12 months on ART treatment by $7.8 \%(n=24)$ and $7.2 \%(n=22)$, respectively. Among children found in the clinical database, $6 / 127$ (4.7\%) had died by the end of follow-up.

\section{Discussion}

The HIV treatment cascades constructed from laboratory data reveal similar outcomes and critical gaps in both Tshwane and uMkhanyakude. For both districts, overall retention in care at the end of the study period was $\sim 33 \%$, with approximately one-third of HIV-infected children having a VL $<1000$ copies $/ \mathrm{mL}$.

After a first positive HIV PCR test, $\sim 75 \%$ of children were presumed to have received confirmatory HIV testing. Alarmingly, within the next 6 months, $\sim 60-70 \%$ of all children with a first positive PCR result were LTFU and only $\sim 50-60 \%$ of those retained achieved a VL $<1000$ copies/mL at 6 months after ART initiation. However, if children remained in care until the first 6-month HIV VL monitoring visit, 73 - 74\% returned for their subsequent visit, with $\sim 57 \%$ achieving a $\mathrm{VL}<1000$ copies $/ \mathrm{mL}$. In spite of similar outcomes in the two districts, the LTFU rates in Tshwane were significantly higher than in uMkhanyakude. Differences may be the result of mobility of urban v. rural infants, particularly where mother-infant pairs return to their rural homes after delivery on account of tradition and cultural norms. ${ }^{[21]}$
The largest gap requiring intervention occurred within 3 months of the first positive HIV PCR test, during which time $\sim 75 \%$ of children returned for HIV-related testing at least once (and half of these children returned a second time) before two-thirds were LTFU within 6 months. By virtue of the HIV-related tests performed at the visit after the first positive PCR test, healthcare workers appeared to have recognised that these children were HIV-exposed. More than $80 \%$ received a PCR or VL test, but it cannot be ascertained whether the healthcare worker had access to the first positive PCR test result and was performing confirmatory early infant diagnosis (EID) testing, or was repeating a screening test unnecessarily. Additionally, the quality of care received at these visits, such as counselling, support, assessment of ART readiness, ART initiation and tracing for defaulters, requires further scrutiny. An understanding of mothers' experiences at the time of positive PCR result return would also be helpful in understanding the high LTFU rates.

Even with birth testing coverage rates in both districts exceeding $80 \%$ in the first year of implementation, more than half $(56 \%)$ of the infants in uMkhanyakude first tested HIV PCR-positive after 3 months of age, in comparison with $41 \%$ of infants in Tshwane. In both districts, infants with a positive PCR test at birth were significantly less likely to be LTFU than infants testing positive at older ages. This is reassuring for a programme that recently introduced birth testing. The $8-10 \%$ of children with a first positive PCR result who were LTFU following discordant PCR and VL results at their return visit should be recognised as being at high risk and monitored closely with follow-up PCR and VL tests to establish a definitive HIV diagnosis.

Approximately 20\% of children retained in care at 6 months subsequently had CD4+ but no VL results at these visits. In these instances, either $\mathrm{VL}$ and $\mathrm{CD} 4+$ monitoring guidelines were not correctly followed, or a specimen for VL could have been submitted by the healthcare worker but not registered by the laboratory, for a variety of reasons. The intervals between VL tests demonstrate that VL monitoring is not performed according to guidelines in high-risk children with VL $>1000$ copies/mL. Urgent intervention is warranted to achieve earlier subsequent VL tests in pursuit of virological control. Clarity around the guideline recommendation of repeating a VL after 6 or 12 months if the 6 -month VL is $<50$ copies $/ \mathrm{mL}$ is required.

A national clinical database that includes laboratory test results is ideal for longitudinal cohort monitoring. Tier.net has the potential to fulfil this function, but even where it is available, it is hampered by incomplete data. Accurate monitoring based on a national laboratory database assumes that HIV monitoring tests are performed as frequently as recommended by guidelines. If not, children retained in clinical care without receiving appropriate HIV testing would be under-represented in the laboratory database. For Tshwane, we demonstrate that $58 \%$ of the study participants were absent from 
the two clinical databases; however, the laboratory database used in isolation missed $7-10 \%$ of children in clinical care but not receiving HIV tests. Combining the clinical and laboratory databases would generate the most accurate treatment cascade. For example, we could ascertain 6/127 (4.7\%) deaths from Tier.net among children misclassified as LTFU from the laboratory-based treatment cascade. Currently it is not possible to comment on deaths using the laboratory cascade, as this metric is not captured in the CDW. However, until data and technical problems involved in making this a reality are resolved, the laboratory database represents the most feasible resource in the medium term for national near-real-time monitoring. This strategy has been used to accurately monitor the national EID programme at a far lower cost than national surveys. ${ }^{[4,5]}$ In addition, since June 2015, laboratory data have been used to generate Results for Action (RfA) reports that are emailed routinely to PMTCT co-ordinators, facility managers and district clinical specialists to enhance follow-up of HIV PCR-positive children. ${ }^{[22]}$ In the light of findings from the present study, RfA dashboards are currently being developed and will also be made available to the above stakeholders to track children who are lost from care and those in care with unsuppressed VLs.

Regarding overall retention in care, similar findings were reported from a cohort of 30 infants initiating ART at a median age of 16 weeks in Johannesburg, SA. Thirty-four percent of HIV-infected infants were retained in care after $>1$ year of ART. ${ }^{[23]}$ Another study conducted in Cape Town, SA, reported a higher rate of retention, 50\% after 2.1 years of ART, in a cohort of infants initiating ART within the first month of life $(n=22) \cdot{ }^{[14]}$ We attribute this high rate of retention in care to the fact that a small sample of children was followed up, and consider that the overall retention rates of $\sim 30 \%$ in our study sites reflect the complexities of managing neonates and infants in HIV care within programmatic settings. Currently, many health facilities lack robust systems for linking and tracking infants in HIV care. The problem is often compounded by a lack of unique patient identifiers for infants. ${ }^{[24]}$ With name changes being common among infants, tracking linkage into care in this group is often difficult, particularly where mother-child pairs alternate between health facilities for care. ${ }^{[15,21,23-25]} \mathrm{In} \mathrm{SA}$, it is common for mother-child pairs to be downreferred to different facilities for primary care after delivery. Mobility of mother-child pairs disrupts co-ordination of care, resulting in patients not linking or remaining in care. Since birth testing is routine practice, it follows that more children are being diagnosed with HIV at very young ages. As a result, facilities require robust systems that can effectively link and track this population while in care. The use of Road to Health booklet identifiers issued with the patient-retained immunisation record at time of birth can go a long way towards achieving this end. ${ }^{[25]}$

Interpretation of findings should take cognisance of the fact that a laboratory database is not equivalent to a clinical longitudinal cohort management system. The laboratory-generated treatment cascade assumes that ART is initiated on all children around the time of the first positive HIV PCR test, which is not the case in practice, particularly for children with discordant HIV confirmatory test results that require further investigation and for those with psychosocial issues and comorbidities. Our findings are limited by the lack of a unique identifier in the NHLS CDW in that the patient-linking algorithm may not have linked all HIV-related tests performed on a particular patient, which could have resulted in under-reporting of retention in care and VL outcomes. Birth HIV PCR tests, associated with poor demographic records, are especially at risk of not being linked to later tests. The latter introduces the potential for overestimating the number of children with a first positive PCR test and age at first diagnosis, and underestimating retention in care. Tshwane is at higher risk of these errors than uMkhanyakude, considering that $30 \%$ of the first positive PCR tests were at birth. We cannot exclude the possibility that children with a first positive PCR test result die before accessing care and therefore never gain entry into a clinical HIV treatment database such as Tier. net. Our data focus only on the public healthcare sector; migration between public and private healthcare is not accounted for, and its magnitude is unknown, but it is more likely in urban areas such as Tshwane. This factor may explain the higher LTFU rates noted in Tshwane.

\section{Conclusions}

We demonstrate the value of using routine laboratory data as a diagnosis, retention in care and VL suppression monitor for HIVinfected children aged $<18$ months, from the initial positive HIV PCR test to identify critical gaps in the programme. This approach to longitudinal cohort monitoring is scalable, can be reported in near realtime and is relatively inexpensive to implement, but is compromised by the absence of a unique identifier and lack of clinical data.

In spite of three-quarters of children returning to care after their first positive PCR result in 2015/16, retention in HIV care thereafter was poor and accounted for the largest gap in LTFU. Twenty percent of children in care had no VL monitoring results and VLs $>1000$ copies/mL were not actively managed, leaving $40 \%$ of children in care at 12 months with a VL $\geq 1000$ copies $/ \mathrm{mL}$. The laboratory database provides an opportunity to begin to improve paediatric HIV services in the country until clinical databases can assume the function of longitudinal cohort monitoring.

\section{Declaration. None.}

Acknowledgements. The authors thank the National Health Laboratory Service for providing laboratory test data, the District Health Services of uMkhanyakude and Tshwane for their hard work managing and co-ordinating PMTCT services in their districts under challenging conditions, and UNICEF and the ELMA Foundation for financial support. Author contributions. GGS, SB and AHM conceived the study design and selected indicators for analysis. GGS, MG and DG acquired the data. FM, AHM, UDF and GGS analysed/interpreted the data. FM, AHM, UDF and GGS wrote the article. UDF, KN, OBM, MG, DG, SB, AHM and GGS provided critical revision and final approval.

Funding. The authors acknowledge financial support for the study from UNICEF South Africa.

Conflicts of interest. None.

\footnotetext{
Kharsany ABM, Quarraisha AK. HIV infection and AIDS in sub-Saharan Africa: Current status, challenges and opportunities. Open AIDS J 2016;10:34-48. https://doi. org/10.2174/1874613601610010034

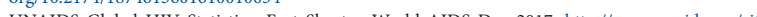
. UNAIDS Global HIV Statistics. Fact Sheet - World AIDS Day 2017. http://w
default/files/media_asset/UNAIDS_FactSheet_en.pdf (accessed 28 October 2018).

default/files/media_asset/UNAIDS_FactSheet_en.pdf (accessed 28 October 2018). . Davies M-A, Pinto J. Targeting 90-90-90 - don't leave the children and adolescents behind. J Int AIDS Soc 2015;18(6):20745. https://doi.org/10.7448/IAS.18.7.20745

4. Sherman GG, Lilian RR, Bhardwaj S, et al. Laboratory information system data demonstrate successful implementation of the prevention of mother-to-child transmission programme in South Africa. S Afr Med J 2014;104(3):235-238. https://doi.org/10.7196/SAMJ.7598

5. Sherman GG, Haeri Mazanderani A, Barron P, et al. Toward elimination of mother-to-child transmission of HIV in South Africa. J Glob Health 2017;7(1):010701. https://doi.org/10.7189/ jogh. 07.010701

6. Moyo F, Haeri Mazanderani A, Barron P, et al. Introduction of routine HIV birth testing in the South African National Consolidated Guidelines. Pediatr Infect Dis J 2018;37(6):559-563. https://doi. org/10.1097/INF.0000000000001840

org/10.1097/N.00000000 7. Horstmann E, Brown J, Islam F, Buck J, Agins BD. Retaining HIV-infected patients in care: Where are we Men Wh 8. McNairy ML, Lamb MR, Carter RL, et al. Retention of HN-infected children on antiretroviral treatment in HIV care and treatment programs in Kenya, Mozambique, Rwanda and Tanzani
J Acquir Immune Defic Syndr 2013;62(3):e70-e81. https://doi.org/10.1097/QAI.0b013e318278bcb0
} 
9. Evans D, Menezes C, Mahomed K, et al. Treatment outcomes of HIV-infected adolescents attending public sector HIV clinics across Gauteng and Mpumalanga, South Africa. AIDS Res Hum Retroviruses 2013;29(6):892-900. https:///doi.org/10.1089/aid.2012.0215

10. Nglazi MD, Kranzer K, Holele P, et al. Treatment outcomes in HIV-infected adolescents attending a Nglazi MD, Kranzer K, Holele P, et al. Treatment outcomes in HIV-infected adolescents attending a
community-based antiretroviral therapy clinic in South Africa. BMC Infect Dis 2012;12(21). https:// doi.org/10.1186/1471-2334-12-21

11. KIDS-ART-LINC Collaboration. Low risk of death, but substantial program attrition, in pediatric HIV treatment cohorts in sub-Saharan Africa. J Acquir Immune Defic Syndr 2008;49(15):523-531. https:// doi.org/10.1097/QAI.0b013e31818aadc

12. Auld AF, Pelletier V, Robin EG, et al. Retention throughout the HIV care and treatment cascade: From diagnosis to antiretroviral treatment of adults and children living with HIV-Haiti, 1985 - 2015. Am J Trop Med Hyg 2017;97(4):57-70. https://doi.org/10.4269/ajtmh.17-0116

13. Abougi LL, Smith C, McFarland EJ. Retention of HIV-infected children in the first 12 months of antiretroviral therapy and predictors of attrition in resource limited settings: A systematic review. PloS One 2016;11(6):e0156506. https://doi.org/10.1371/journal.pone.0156506

14. Frigati L, Wynberg E, Maritz J, Holgate S, Cotton MF, Rabie H. Antiretroviral therapy initiated in the first month of life. Pediatr Infect Dis I 2017;36(6):584-587. https://doi.org/10.1097/INF.0000000000001504

15. National Department of Health, South Africa. National Consolidated Guidelines for the Prevention of Mother-to-Child Transmission of HIV (PMTCT) and the Management of HIV in Children, Adolescents and Adults. Pretoria: NDoH, 2015, http://www.sahivsoc.org/Files/ART\%20 Guidelines\%2015052015.pdf (accessed 15 October 2018).

16. Mazanderani AH, Moyo F, Kufa T, Maritz J, Sherman GG. Differentiating clearly positive from indeterminate results: A review of irreproducible HIV-1 PCR positive samples from South Africass Early Infant Diagnosis Program, 2010 - 2015. Diagn Microbiol Infect Dis 2018;91(3):248-255. https:// Early Infant Diagnosis Program, 2010 - 2015.
doi.org/10.1016/j.diagmicrobio.2018.02.019

17. Mazanderani AH, Moyo F, Kufa T, Maritz J, Sherman GG. Declining baseline viraemia and 7. Mazanderani AH, Moyo F, Kufa T, Maritz J, Sherman GG. Declining baseline viraemia and
escalating discordant HIV-1 confirmatory results within South Africas Early Infant Diagnosis Program, 2010 - 2016. J Acquir Immune Defic Syndr 2018;77(2):212-216. https://doi.org/10.1016/j. diagmicrobio.2018.02.019
18. Technau KG, Mazanderani AH, Kuhn L, et al. Prevalence and outcomes of HIV-1 diagnostic challenges during universal birth testing - an urban South African observational cohort. J Int AIDS Soc 2017;20(6):21761. https://doi.org/10.7448/IAS.20.7.21761

19. Statistics South Africa. Recorded live births 2013 - 2015. Statistical release P0305. Pretoria: Stats SA 2016. http://www.statssa.gov.za/publications/P0305/P03052015.pdf (accessed 19 July 2018).

20. National Department of Health, South Africa. 2015 National antenatal sentinel HIV \& syphilis surver report. http://www.health.gov.za/index.php/shortcodes/2015-03-29-10-42-47/2015-04-30-08-1810/2015-04-30-08-21-56?download $=2584: 2015$-national - antenatal-hiv-prevalence- - survey-final 23 oct17 (accessed 31 October 2018).

21. Clouse K, Vermund SH, Maskew M, et al. Mobility and clinic switching among postpartum women considered lost to HIV care in South Africa. J Acquir Immune Defic Syndr 2017;74(4):383-389. https:// doi.org/10.1097/QAL.0000000000001284

22. Moyo F, Haeri Mazanderani A, Bhardwaj S, et al. Near-real-time tracking of gaps in the prevention of mother-to-child transmission of HIV in three districts of KwaZulu-Natal Province, South Africa. S Afr Med J 2018;108(4):319-324. https://doi.org/10.7196/SAMJ.2017.v108i4.12630

23. Lilian RR, Kalk E, Technau KG, Sherman GG. Birth diagnosis of HIV infection in infants to reduce infant mortality and monitor for elimination of mother-to-child transmission. Pediatr Infect Dis J 2013:32(10):1080-1085. https://doi.org/10.1097/INF.0b013e318290622e

24. Macleod W, Bor I, Crawford K, Carmona S. Analysis of big data for better targeting of ART adherence strategies: Spatial clustering analysis of viral load suppression by South African province, district sub-district and facility (April 2014 - March 2015). 2015. https://openknowledge.worldbank.org handle/10986/25399 (accessed 31 October 2018).

25. Haeri Mazanderani A, Sherman GG, Goga AE, Moyo F, Feucht U. Leveraging the road to healt booklet as a unique patient identifier to monitor the prevention of mother to child transmissio programme. S Afr Med J 2018;108(9):729-733. https://doi.org/10.7196/SAMJ.2018.v108i9.13093

Accepted 5 March 2019. 